\title{
JAK Inhibition: A new therapeutic strategy for management of chronic diseases
}

\author{
Hao $\mathbf{Y u}^{1 *}$, Etty N Benveniste ${ }^{2}$ and Hongwei Qin² \\ ${ }^{1}$ Department of Epidemiology, University of Texas, MD Anderson Cancer Center, Houston, TX 77030, USA \\ ${ }^{2}$ Department of Cell, Developmental and Integrative Biology, University of Alabama at Birmingham, Birmingham, AL 35294, USA
}

Aberrant activation of the Janus Kinase (JAK) pathway has been reported in a variety of disease states, including inflammatory conditions, hematologic malignancies, and solid tumors [1]. Preclinical evidence suggests that investigational drugs that target this pathway may provide therapeutic benefit for some disorders. Thus, improving our understanding of this pathway and how it is regulated could contribute to the development of new therapeutic strategies for targeting a broad spectrum of acute and chronic conditions.

JAK pathway signaling can be initiated by numerous cytokines and has been shown to be required for initiating innate immune responses, orchestrating adaptive immunity, and ultimately constraining immune responses. The JAK family is comprised of four members: JAK1, JAK2, JAK3, and TYK2. These receptor-associated kinases are activated by conformational changes in their respective receptors induced by ligand binding. Several recent studies have extended the repertoire of factors that can promote activation of JAK / STAT signaling to include Tolllike receptors (TLRs), such as TLR9 and TLR4, microRNAs (miRNAs), and several G-protein-coupled receptors (GPCRs) [2]. Upon activation, phosphorylation on specific tyrosine residues converts inactive JAKs into catalytically active tyrosine kinases. Activated JAKs phosphorylate tyrosine residues in the cytoplasmic region of the receptor to create binding sites for signal transducers and activators of transcription (STATs). JAK-mediated STAT phosphorylation promotes the formation of stable homodimers or heterodimers that are translocated into the nucleus. Once in the nucleus, STAT dimers bind specific promoter elements, termed interferon-gamma activated sequences (GAS) or interferon-stimulated response elements (IRSE), to induce transcription of interferon-stimulated genes (ISG), which can regulate many cellular processes, including proliferation, differentiation, and apoptosis [3].

Cytokines are critical for regulation of the development, differentiation, and function of myeloid cells and $\mathrm{T}$ cells. Thus, inhibition or hyper activation of the JAK / STAT pathway may have therapeutic benefit for several immune-mediated chronic diseases [4]. In $50 \%$ to $60 \%$ of patients with primary myelofibrosis, a gain-offunction acquired somatic mutation is present in the JAK2 gene. The JAK2 V617F mutation arises from a single base $\mathrm{G}$ to $\mathrm{T}$ transversion in the pseudokinase domain of JAK2, which results in a valine to phenylalanine substitution at codon 617. This substitution has been reported to disrupt the auto-inhibitory activity of the pseudokinase domain (JH2), thus constitutively activating the kinase domain (JH1) [5]. The identification of inappropriate JAK/STAT activation in this disorder has led to the investigation of specific JAK1/2 inhibitors, one of which, Ruxolitinib, has been assessed in phase 3 trials [5].

JAK/STAT signaling has also been shown to play critical roles in cancer cell proliferation and survival, tumor immunosuppression, angiogenesis, and metastasis of solid tumors. Excessive JAK / STAT activity has been found in tumor tissue samples derived from a large number of patients across a range of tumor types, suggesting that JAK/STAT inhibitors may be effective chemotherapeutic agents [3]. Consistent with this hypothesis, in a preclinical study, AZD1480, an ATP-competitive inhibitor of JAK1/2, was found to inhibit the growth of subcutaneous tumors and increase survival of mice bearing intracranial glioblastoma tumors [6]. The efficacy of AZD1480 was also examined in IL-6-driven breast, ovarian and prostate cancer models, resulting in suppression of tumor growth [7]. JAK inhibitors are now under investigation in phase I clinical trials for solid tumors [7].

JAK inhibitors have also been explored as treatments for autoimmune diseases, including multiple sclerosis, arthritis, psoriasis, and inflammatory bowel disease. In experimental autoimmune encephalomyelitis (EAE), a rodent model of multiple sclerosis, treatment with AZD1480 decreases disease severity by inhibiting JAK / STAT activation in the brain and reducing expression of proinflammatory cytokines and chemokines [4]. In rheumatoid arthritis patients, Tofacitinib, a JAK inhibitor, has been shown to improve joint symptoms and reduce joint damage in phase 3 trials [8]. Similarly, clinical trials of oral and topical administration of Tofacitinib for psoriasis have shown statistically significant improvements in symptoms compared to placebo [9].

Although JAK inhibition is a promising therapeutic strategy for many immune-related disorders, unfortunately, JAK inhibitors are also associated with an increased risk of certain cancers, high cholesterol, triglycerides, liver function abnormalities, kidney dysfunction, and decreased white and red blood cell counts [7]. Because JAKs are required for normal hematopoiesis, JAK inhibition results in anemia and thrombocytopenia unless different dosing schedules or lower doses are used [8]. The challenge in inhibiting JAKs for the treatment of chronic disease lies in the fact that cells employ a highly diverse range of strategies to activate the JAK pathway. Therefore, it is nearly impossible to effectively manage chronic conditions using JAK inhibitors without impairing normal cellular functions. Furthermore, given the high similarity of ATP-binding pockets among the $\sim 500$ distinct kinases in humans, new targeting approaches are needed to increase selectivity and specificity of JAK inhibitors [7]. However, whether more selective JAK inhibitors will demonstrate improved therapeutic benefit remains to be determined.

In recent years, major progress has been made in understanding the cellular mechanisms that regulate JAK / STAT signaling, which could lead to novel therapeutic strategies to selectively regulate this pathway. Approximately 50 miRNAs have been predicted to negatively

*Corresponding author: Hao Yu, Ph.D, Department of Epidemiology, University of Texas MD Anderson Cancer Center, Houston, TX 77030, United States, Tel: +1-713-832-3852; E-mail: haoyu1h@gmail.com

Received April 22, 2016; Accepted April 22, 2016; Published April 27, 2016

Citation: Yu H, Benveniste EN, Qin H (2016) JAK Inhibition: A new therapeutic strategy for management of chronic diseases. J Biomol Res Ther 5: e148. doi:10.4172/2167-7956.1000e148

Copyright: (c) $2016 \mathrm{Yu} \mathrm{H}$, et al. This is an open-access article distributed under the terms of the Creative Commons Attribution License, which permits unrestricted use, distribution, and reproduction in any medium, provided the original author and source are credited. 
Citation: Yu H, Benveniste EN, Qin H (2016) JAK Inhibition: A new therapeutic strategy for management of chronic diseases. J Biomol Res Ther 5: e148. doi:10.4172/2167-7956.1000e148

Page 2 of 2

regulate the JAK / STAT pathway, and of these miRNAs, let-7, miR20a, and miR-93 have been directly validated in cancer cells [2]. Thus, microRNA mimics are currently under clinical development for treatment of chronic diseases, and some promising agents have demonstrated clinical proof-of-concept.

As a result of recent immunological studies, it is now recognized that JAKs are also negatively regulated by three major classes of regulators in addition to miRNAs: suppressors of cytokine signaling (SOCS), protein inhibitors of activated stats (PIAS), and protein tyrosine phosphatases (PTP) [10]. In particular, SOCS3 has been shown to bind and directly inhibit the catalytic domains of JAKs via an evolutionarily conserved motif. Taking advantage of this role, a preclinical study has revealed that expression of SOCS3 in myeloid cells provides protection through deactivation of neuroinflammatory responses [11]. In non-small cell lung cancer (NSCLC) tumor samples, expression of SOCS3 has been shown to be lost due to promoter hyper methylation, an epigenetic change that reduces gene transcription. The impact of this decrease in expression on JAK / STAT pathway activation was confirmed in an NSCLC cell line, in which restoration of SOCS3 expression was shown to reduce constitutive STAT3 phosphorylation [12]. Similarly, in a prostate tumor model, targeted loss of SOCS3 in myeloid cells significantly enhanced tumor growth via regulation of myeloid-derived suppressor cells (MDSC) development and function [13]. Finally, an exciting discovery at the Walter and Eliza Hall Institute led to the identification of a novel, ATP-independent mechanism by which SOCS3 inhibits JAK catalytic activity [14]. Compared with the competitive ATP-mimetic that is currently used to inhibit JAK activity, non-competitive kinase inhibitors are highly desirable and have a selective advantage because they cannot be out-competed by endogenous ATP [15]. Therefore, greater specificity of JAK inhibition can be achieved with these inhibitors with reduced undesirable, offtarget effects. As such, further development of this technology will likely lead to the emergence of a new class of highly specific JAK inhibitors.

Based on the current state of the field, it is not difficult to imagine that in the next decade, there will be many breakthroughs in our knowledge and understanding of immune-mediated chronic diseases. Importantly, through the use of these newly developed JAK inhibition strategies, negative regulation of the JAK pathway may provide new therapeutic avenues for management of chronic disease.

\section{References}

1. Yu H, Pardoll D, Jove R (2009) STAT's in cancer inflammation and immunity: a leading role for STAT3. Nat Rev Cancer 9: 798-809.

2. Yu H, Lee H, Herrmann A, Buettner R, Jove R (2014) Revisiting STAT3 signalling in cancer: new and unexpected biological functions. Nat Rev Cancer 14: $736-46$.

3. Inagaki-Ohara K, Kondo T, Ito M, Yoshimura A (2013) SOCS, inflammation, and cancer. Jak-Stat 2: e24053.

4. Liu Y, Holdbrooks AT, De Sarno P, Rowse AL, Yanagisawa LL, et al. (2014) Therapeutic efficacy of suppressing the Jak / STAT pathway in multiple models of experimental autoimmune encephalomyelitis. J Immunol 192: 59-72.

5. Ostojic A, Vrhovac R, Verstovsek S (2012) Ruxolitinib for the treatment of myelofibrosis: its clinical potential. Ther Clin Risk Manag 8: 95-103.

6. McFarland BC, Ma JY, Langford CP, Gillespie GY, Yu H, et al. (2011) Therapeutic potential of AZD1480 for the treatment of human glioblastoma. Mol Cancer Ther 10: 2384-93.

7. O'Shea JJ, Schwartz DM, Villarino AV, Gadina M, Mclnnes IB, et al. (2015) The JAK-STAT pathway: impact on human disease and therapeutic intervention Annu Rev Med 66: 311-28.

8. Lee EB, Fleischmann R, Hall S, Wilkinson B, Bradley JD, et al. (2014) Tofacitinib versus methotrexate in rheumatoid arthritis. N Engl J Med 370: 2377-86.

9. Papp KA, Menter MA, Abe M, Elewski B, Feldman SR, et al. (2015) Tofacitinib an oral Janus kinase inhibitor, for the treatment of chronic plaque psoriasis: results from two randomized, placebo-controlled, phase III trials. $\mathrm{Br}$ J Dermatol 173: 949-61.

10. Baker BJ, Akhtar LN, Benveniste EN S(2009) OCS1 and SOCS3 in the contro of CNS immunity. Trends Immunol 30: 392-400.

11. Qin H, Yeh WI, De Sarno P, Holdbrooks AT, Liu Y, et al. (2012) Signal transducer and activator of transcription-3/suppressor of cytokine signaling-3 (STAT3 / SOCS3) axis in myeloid cells regulates neuroinflammation. Proc Natl Acad Sci U S A 109: 5004-9.

12. He B, You L, Uematsu K, Zang K, Xu Z, et al. (2003) SoCS-3 is frequently silenced by hypermethylation and suppresses cell growth in human lung cancer. Proc Natl Acad Sci U S A 100: 14133-8.

13. Yu H, Liu Y, McFarland BC, Deshane JS, Hurst DR, et al. (2015) SOCS3 Deficiency in Myeloid Cells Promotes Tumor Development: Involvement of STAT3 Activation and Myeloid-Derived Suppressor Cells. Cancer Immunol Res 3: 727-40.

14. Babon JJ, Kershaw NJ, Murphy JM, Varghese LN, Laktyushin A, et al. (2012) Suppression of Cytokine Signaling by SOCS3: Characterization of the Mode of Inhibition and the Basis of Its Specificity. Immunity 36: 239-50.

15. Kershaw NJ, Murphy JM, Liau NP, Varghese LN, Laktyushin A, et al. (2013) SOCS3 binds specific receptor-JAK complexes to control cytokine signaling by direct kinase inhibition. Nat Struct Mol Biol 20: 469-76. 